\title{
Insulation lifetime improvement of polyimide thin film neural implants.
}

\author{
Frederik Ceyssens and Robert Puers
}

\begin{abstract}
Objective. This work deals with studying and improving the insulation lifetime of polyimide-insulated thin film neural implants, or related polyimide-based medical implants. Approach. The evolution of the leak impedance of insulated conductors was investigated in saline water at $40{ }^{\circ} \mathrm{C}$. The fabrication process as commonly found in literature for polyimide/platinum/polyimide microelectrode arrays was compared with three possible improvements: one based on lowering the curing temperature of the lower layer, one based on chemical activation and one based on an additional plasma activation step. Main results. The lower curing temperature process was found to yield a 7.5-fold improved lifetime compared with the state of the art process. Also, the leak impedances found after soak testing are an order of magnitude higher compared to the standard process. Significance. By improving the lifetime and insulation impedance of polyimide insulation with one order of magnitude, this work increases the applicability of polyimide in chronic thin film neural considerably.
\end{abstract}

\section{Keywords}

$E C o G, \mu E C o G$, neural electrode array, polyimide, thin film electrode array, lifetime testing, neural electrode, flexible.

\section{Introduction}

High density neuroelectronic interfaces have been under development for research and clinical use for several decades. An important class of such interfaces is based on needle-shaped electrodes that penetrate the brain and are thus able to approach the neuron soma closely, allowing to record individual action potentials [1].

Another important class is formed by the so-called thin film neural electrode arrays which are often placed directly on the cortex or the meninges. Often, polyimide is used a base material for these flexible implants. Though the recorded local field potentials (LFPs) are the superposition of the signals of many hundreds of neurons, they are still attractive as they are more stable [2] than action potentials coming from a single neuron. Also, the lower invasiveness promises a better long-term bioacceptance. Such electrode arrays have applications in i.a. $\mu E C o G$ (microElectroCorticoGraphic) recording [3-7], retinal stimulation [7] and experimental cochlear implants [8]. Also, the polyimide-based die-level packaging technologies investigated for certain experimental ultra-thin implantable medical devices are very similar [10]. 
In light of the trend towards chronic monitoring over several months to years [11-13], assessing and improving the lifetime of implants becomes very important. Aspects of electrode lifetime include biofouling or encapsulation by the immune system [12], damage to or adhesion loss of the electrode's conductor material [14] and failure of the insulation layer $[15,16,17]$. This article focuses on the latter aspect.

In current work on thin film electrode arrays, Parylene $\mathrm{C}$ and polyimide (PI) are the most prevailing insulation layers.

Recent work has shown that the lifetime of the insulation of Parylene-based implants can be drastically improved by post-fabrication annealing (i.e. recrystallization) of the Parylene at temperatures between 200 and $300^{\circ} \mathrm{C}$ in a $\mathrm{N}_{2}$ atmosphere $[15,16,17]$. Such recrystallization is not possible with $\mathrm{PI}$, which is already cured at temperatures up to $400^{\circ} \mathrm{C}$ during fabrication and degrades around $550^{\circ} \mathrm{C}$. As elaborated in the discussion section, the superior mechanical properties of $\mathrm{PI}$ are an incentive to improve the lifetime of PI thin film insulation to the same level.

In this article, we will investigate if techniques other than annealing can yield a significant lifetime improvement for the PI insulation layer of thin film neural implants. In particular, we will investigate the use of a lower curing temperature for the lower PI layer. This would leave an increased number of reactive groups that can bond covalently with the top PI layer, which is cured at the normal high temperature. Another investigated alternative adds plasma activation of the lower PI layer. In another alternative process, the $\mathrm{PI}$ is chemically activated.

\section{Experimental design}

\subsection{Design of test structures}

A test structure was designed for lifetime testing by immersion in saline solution. The design (figure 1) features an $18 \mathrm{~mm}$ long shaft, containing 16 conductive platinum paths $25 \mu \mathrm{m}$ wide, spaced $25 \mu \mathrm{m}$ apart. The conductive paths are squeezed between two insulation layers. Half of the paths end blindly, the other half end on a $41 \mu \mathrm{m}$ diameter opening in the upper isolation layer, forming an electrode in contact with the medium outside. Both types are placed alternatingly. This configuration ensures that any delamination defect would create a leak path to the outside medium independent on the position. Furthermore, this alternation produces a more realistic test that also includes delamination starting from the platinum-PI interface in contact with saline solution. The closest designed distance between the edge of a contact hole and the neighboring blind conductor is $17 \mu \mathrm{m}$. This is a relatively strong test for this failure mode, as in many implant designs the distance between a contact hole and nearest conductor can be made larger. Other potential failure modes could be based on diffusion of water through the insulating material and subsequent nucleation at the interface, or be initiated from the interface between the two insulation layers from the side of the test structure (figure 1, bottom center). On figure 1, it is also noticeable that the etch holes towards the electrodes are designed with a $5 \mu \mathrm{m}$ smaller radius than the electrodes themselves. This way, an anisotropic reactive ion etch will stop on the $\mathrm{Pt}$ of the electrodes without damaging the lower PI layer, which results in a significant tolerance for 
overetching. This allows a single reactive ion etch step to be used both to open the electrodes (which requires a $3.5 \mu \mathrm{m}$ deep etch) as well as to define the contours of the devices for which a $7 \mu \mathrm{m}$ deep etch is needed to reach the supporting substrate.

\subsection{Fabrication of test structures}

Four different sets of test structures (PI-1, PI-2, PI-3 and PI-4) were fabricated, each using a variant of a two-mask, three-layer lithographic process. The fabrication processes are detailed out in the supplementary data section. The fundamental differences are summarized in Table 1 and described below.

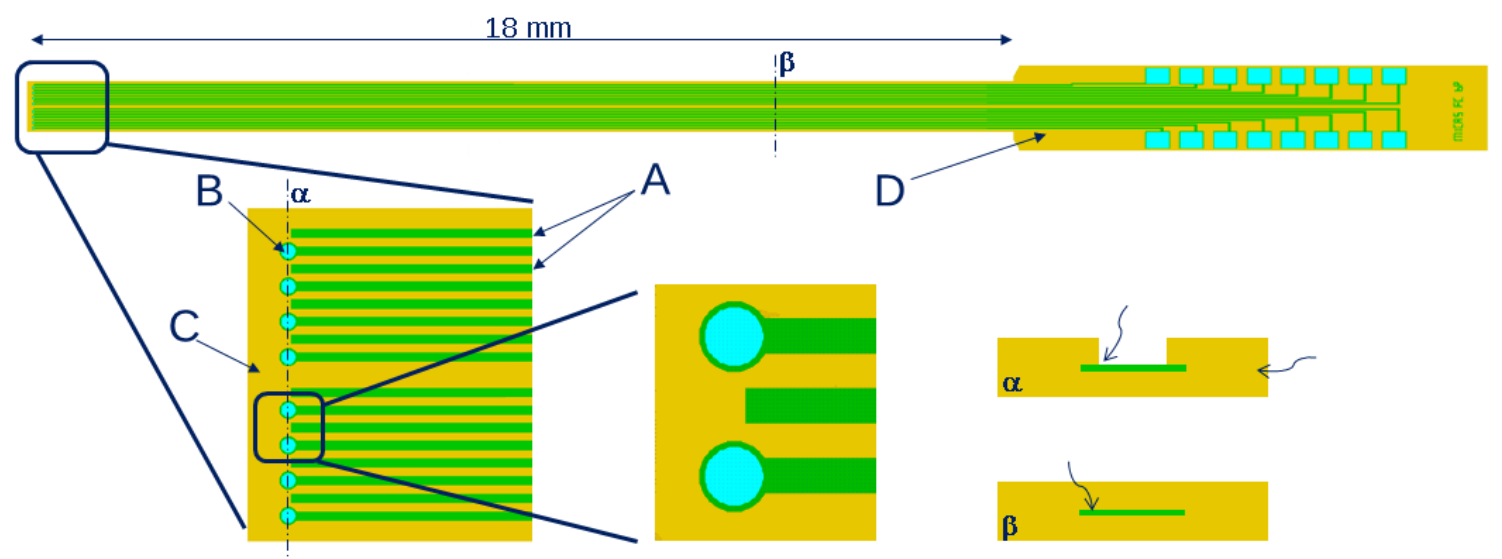

Figure 1: design of test structure. A: blind ending conductive paths between which the impedance is measured ("channels"). B: conductive path ending in a $41 \mu \mathrm{m}$ diameter electrode. C: insulation layers under and on top of conductive path. D: connector part with bond pads.

Cross sections $\alpha$ and $B$ show the layered build-up. Curved arrows indicate possible failure mechanisms:

delamination from the Pt-insulation and insulation-insulation interface and water intrusion trough the insulation

PI-1 samples were made with HD Microsystems PI-2611 polyimide based on the standard process such as found in on our earlier work [17]. Processes that are similar with respect to the curing of the polyimide have been used by many other authors as well $[5,6,7,8]$. In this $\mathrm{PI}-1$ process, the lower $\mathrm{PI}$ is fully cured at $340^{\circ} \mathrm{C}$ or above before depositing the conductors.

The $130 \mathrm{~nm}$ thick Pt conductors are deposited by sputter coating and patterned using a liftoff method based on a dual layer of LOR1OB and S1818 photoresist with a release step in NMP (n-methyl-2pyrrolidone) at room temperature. Then, the second $\mathrm{PI}$ layer is deposited and fully cured at $340^{\circ} \mathrm{C}$ as well. The structures are then patterned using Reactive Ion Etching (RIE) with an aluminum mask, and released from the carrier wafer. The arrays fabricated are $7 \mu \mathrm{m}$ thick in total. The thickness is a factor 2 to 3 lower than typically used in literature, as the developed fabrication process is part of a project aiming at highly flexible, minimally irritating implants. An example test structure is shown in figure 2.

For the PI-2 samples, the curing temperature of the lower PI layer was lowered in order to produce a layer with a higher reactivity to the second PI layer. The curing temperature was first set to $170^{\circ} \mathrm{C}$. However, the lower PI layer did not seem to be undamaged by the subsequent liftoff step in NMP used 
for the patterning of the Pt layer: relatively large, approximately $300 \mathrm{~nm}$ thick flakes came off. Therefore, the curing temperature was raised to $205^{\circ} \mathrm{C}$. With this temperature no damage was seen after the liftoff step. No further temperature optimization was performed.

For the fabrication of the PI-3 samples, the protocol of PI-2 was followed except that the deposition of the second PI layer was directly preceded by a plasma activation step of the lower PI layer (plasma etching for $30^{\prime \prime}$ at $50 \mathrm{~W}, 150 \mathrm{mTorr}$ pressure, $40 \mathrm{sccm} \mathrm{O}$ flow and $20^{\circ} \mathrm{C}$ temperature, using a PT etcher with $125 \mathrm{~mm}$ diameter platen electrode). Plasma activation is a common procedure which is often helpful in increasing the adhesion of i.a. metals deposited on polymers [19].

For the fabrication of the PI-4 samples, a chemical activation procedure [20] was used instead of plasma. This procedure breaks open the PI chain to insert additional carboxylic acid groups as illustrated in figure 3. These groups will react in condensation reactions at the elevated temperatures the sample encounters when the second PI layer is cured, creating additional covalent bonds between the two layers.

The activation procedure is applied right before the deposition of the second PI layer, and goes as follows:

- Soak 10 minutes in $0.1 \mathrm{M} \mathrm{NaOH}\left(20^{\circ} \mathrm{C}\right)$

- Rinse in a stream of ultrapure water for 1 minute

- Soak for 20 minutes in $0.1 \mathrm{M}$ acetic acid $\left(20^{\circ} \mathrm{C}\right)$

- $\quad$ Rinse in a stream of ultrapure water for 5 minutes

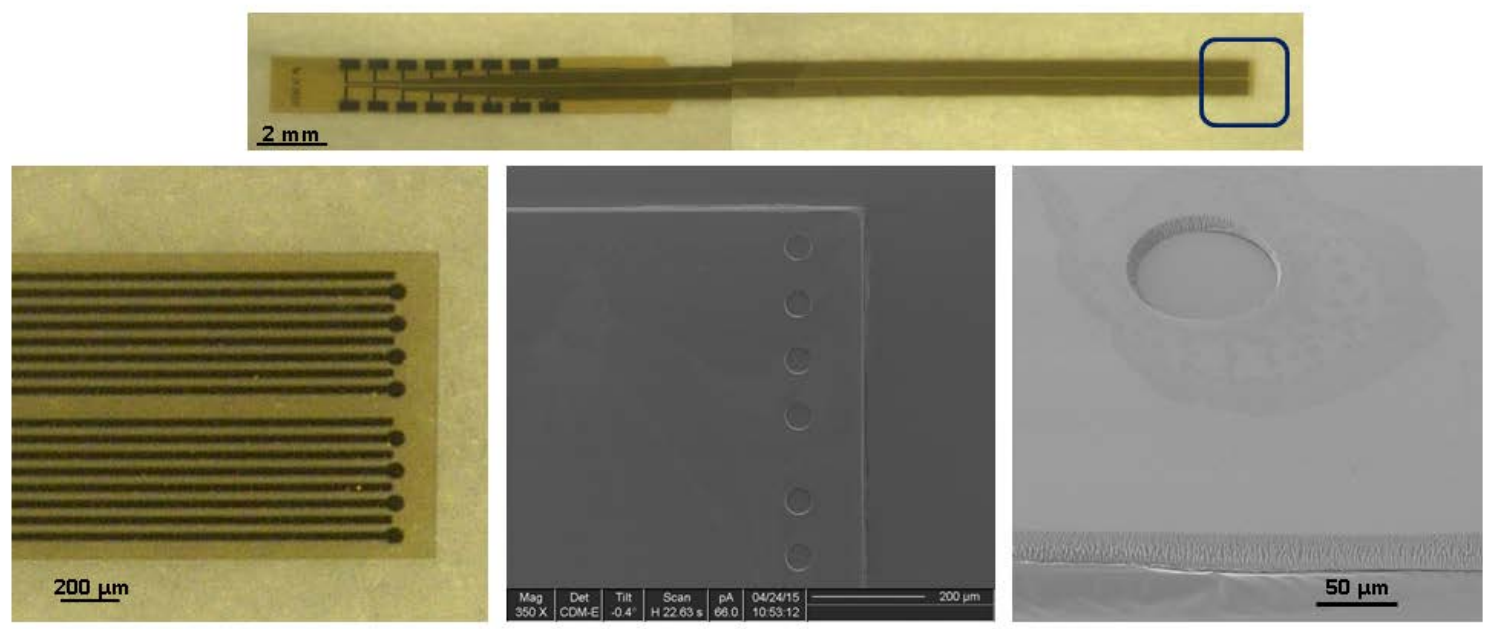

Figure 2: Optical microscope and Focused lonBeam (FIB) pictures of fabricated PI test structure. The two etch depths obtained with a single fabrication step as described in section 2.1 are clearly visible in the lower right picture. 
<smiles>Cc1ccc(N2C(=O)c3ccc(-c4ccc5c(c4)C(=O)N(C)C5=O)cc3C2=O)cc1</smiles><smiles>CNC(=O)c1cc(-c2ccc(C(=O)O[Na])c(C(=O)Nc3ccc(C)cc3)c2)ccc1C(=O)O[Na]</smiles><smiles>CNC(=O)c1cc(-c2ccc(C(=O)O)c(C(=O)Nc3ccc(C)cc3)c2)ccc1C(=O)O</smiles>

Figure 3: chemical activation procedure.

\begin{tabular}{|l|l|l|l|l|}
\hline & $\begin{array}{l}\text { PI-1: Standard } \\
\text { PI process }\end{array}$ & $\begin{array}{l}\text { PI-2: Adapted } \\
\text { PI process }\end{array}$ & $\begin{array}{l}\text { PI-3: Adapted PI } \\
\text { process + plasma }\end{array}$ & $\begin{array}{l}\text { PI-4: chemical } \\
\text { activation }\end{array}$ \\
\hline Insulation I & Cured at $340^{\circ} \mathrm{C}$ & Cured at $205^{\circ} \mathrm{C}$ & Cured at $205^{\circ} \mathrm{C}$ & Cured at $205^{\circ} \mathrm{C}$ \\
\hline Insulation II & Cured at $340^{\circ} \mathrm{C}$ & Cured at $340^{\circ} \mathrm{C}$ & $\begin{array}{l}\text { activation of } \\
\text { substrate } \\
\text { - Cured at } 340^{\circ} \mathrm{C}\end{array}$ & $\begin{array}{l}\text { - Chemical activation } \\
\text { of substrate in } 0.1 \mathrm{M} \\
\text { NaOH. }\end{array}$ \\
\hline & & & Cured at $340^{\circ} \mathrm{C}$ \\
\hline
\end{tabular}

Table 1: Essential differences between the investigated fabrication processes. 


\section{Lifetime testing}

\subsection{Mechanical adhesion test}

Before release from the carrier wafer, four structures of each kind investigated were subjected to a simple mechanical peel test. All samples passed this test except the PI-3 samples, of which the top PI layer could be easily detached from the lower PI layer. Therefore, it was concluded that an oxygen plasma treatment of the lower PI layer is detrimental for adhesion. The PI-3 samples were not further used in the long-term soak tests.

A possible explanation for this is the further oxidation of the anhydride groups in the $S$ Biphenyldianhydride (BPDA) and/or of the amine groups in the p-Phenylenediamine (PDA) the PI2611 is composed of by the plasma, lowering reactivity with a second PI2611 layer (figure 4).

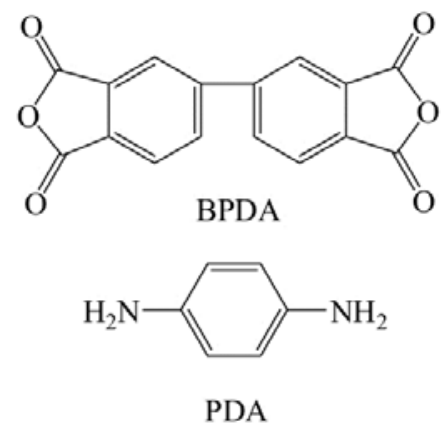

Figure 4: Composition of PI-2611 polyimide (supplier's data).

\subsection{Mounting in test setup}

The connector part of the test structure was placed on a glass substrate and wires were attached on the bond pads using Epo-Tek H2OE conductive epoxy. A layer of Epo-Tek H54 high temperature epoxy was used to insulate and attach the connector and wires. The connector assembly was mounted in the neck of a plastic bottle with the shaft of the test structure facing down. The bottle was filled with phosphate buffered saline (PBS) with a pH value of 7.2. The composition of the PBS was: $8 \mathrm{~g} / \mathrm{L} \mathrm{NaCl}, 0.2 \mathrm{~g} / \mathrm{L} \mathrm{KCl}$, $1.44 \mathrm{~g} / \mathrm{L} \mathrm{Na}_{2} \mathrm{HPO}_{4}$ and $0.24 \mathrm{~g} / \mathrm{L} \mathrm{KH}_{2} \mathrm{PO}_{4}$. The shaft of the test structure was lowered approximately 15 $\mathrm{mm}$ into the PBS. A loosely wound metal coil was placed around the test structure shaft in the water to keep it from floating, e.g. because of air bubbles attaching to it. The PBS was heated to $40^{\circ} \mathrm{C}$ throughout the experiment. Parafilm was used to cover the bottle. Water evaporation was compensated for by adding demineralized water when needed. The impedance measuring device was a Keithley 2612A sourcemeter, connected to the channels through a relay-based switch.

\subsection{Soak test:}

In order to assess the long-term performance of the insulation layers, a soak test was done in PBS at $40^{\circ} \mathrm{C}$ in the setup described in section 3.2. At least seven channels produced by each of the fabrication processes that passed the mechanical adhesion test (PI-1, $\mathrm{PI}-2$ and $\mathrm{PI}-4)$ were soak tested at $40^{\circ} \mathrm{C}$. 
In regular intervals, the DC impedance between a of blind-ending conductor as shown in figure 1 (called "channels" from now on) and a large external reference electrode was measured. The source meter was used in current-sensing mode with an applied voltage of $2 \mathrm{~V}$. Measuring time was 4 seconds per channel, and sample interval was one minute for each channel. The measured impedances were put through a moving average filter to remove occasional spikes. The experiment was run over 7 weeks, unless all channels had failed earlier.

The measured cumulative failure graph, lifetimes and standard deviations are plotted in figure 5 . The raw impedance data as a function of time are given in the supplementary data section in figures S2 and S3.

The chosen failure criterion was a drop of the DC impedance under $50 \mathrm{M} \Omega$ for the first time - as can be seen in figures S2 and S3, the impedance behaves stochastically and can go up above the threshold again after it has been reached. The $50 \mathrm{M} \Omega$ criterion is reasonable as it is one order of magnitude above the DC impedance of the electrodes used in our work [17]. Also, note that, the measured DC impedance between a large external electrode in the PBS and an open electrode in the test structure was $25 \mathrm{M} \Omega$ at the start of the experiment.

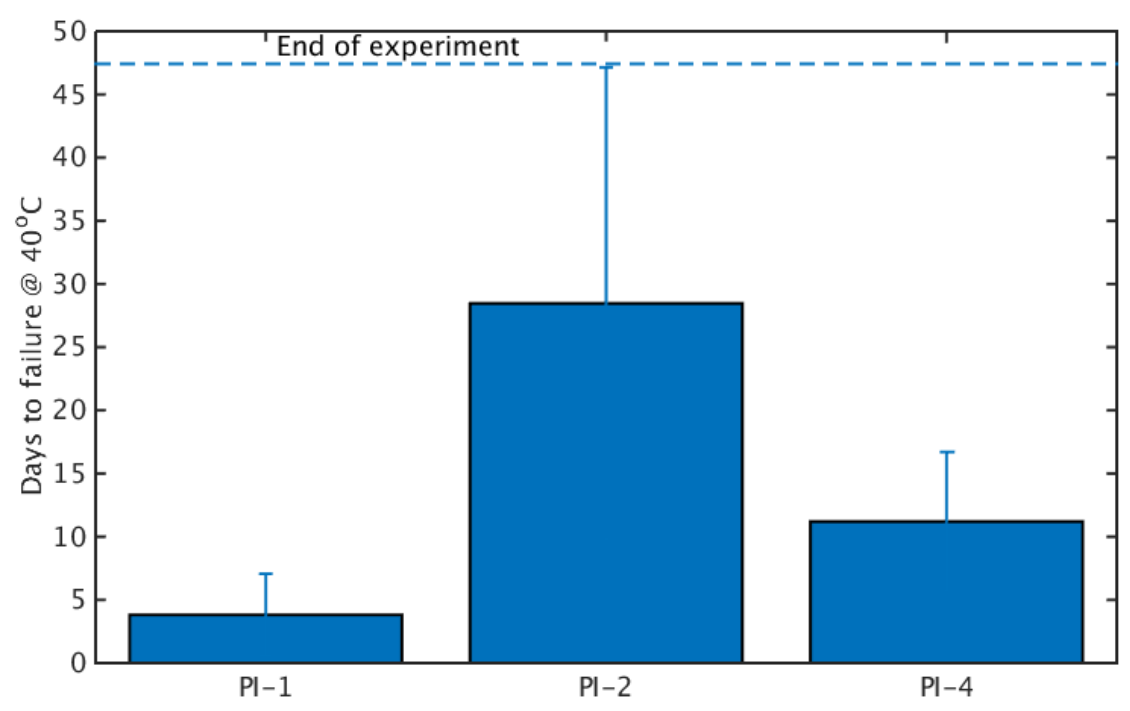




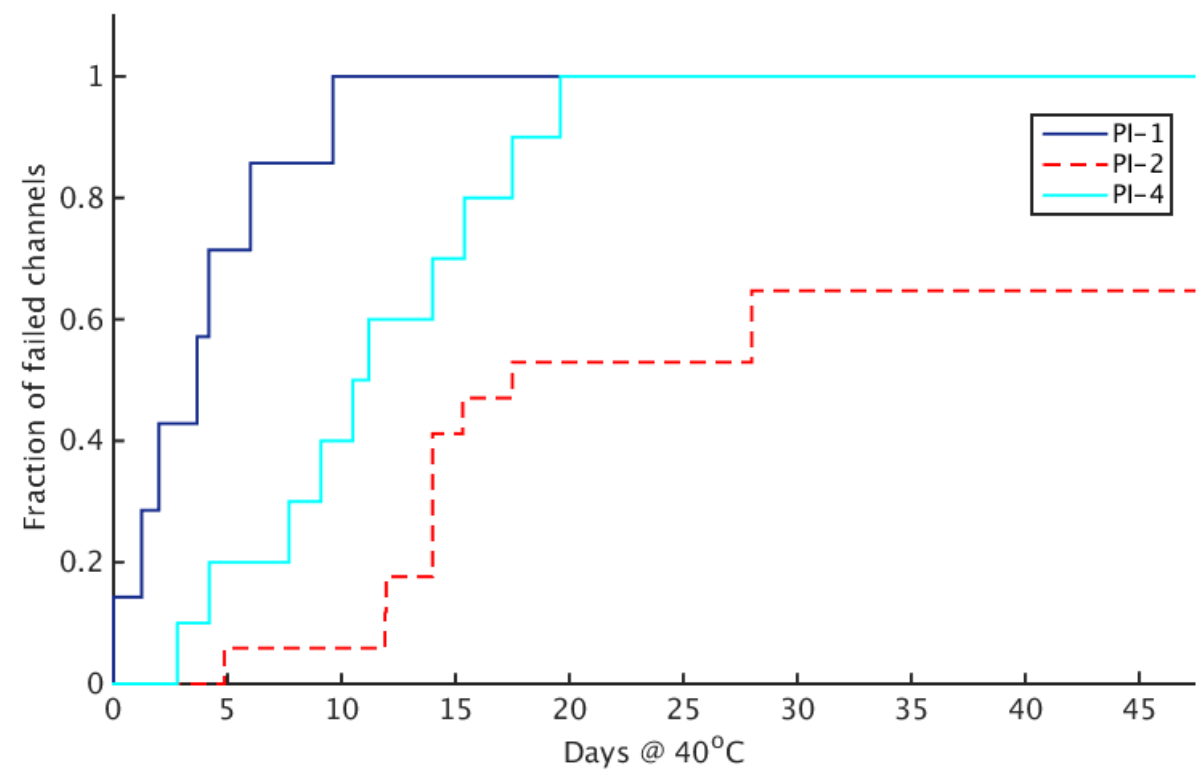

Figure 5: results of soak test at $40^{\circ} \mathrm{C}$.Top: bar graph showing average lifetimes and standard deviation. Bottom: cumulative failure graph for the different processes. Because of not passing the mechanical adhesion test, PI-3 samples were not tested further.

The average time to failure of a channel of each type of test sample is shown in table 2. This table also shows the average leak impedance in the failed channels that can be expected at the end of the experiment (including the non-failed channels of PI- 2 would increase this drastically). It can be seen that the adapted $\mathrm{PI}-2$ process improves lifetime at $40^{\circ} \mathrm{C}$ in saline water with a factor of 7.5 compared to the classic PI-1 process. This is even an underestimation, as one third of the PI-2 samples had not failed at the end of the experiment. The PI-4 process is about three times better in terms of lifetime, which could be because of the lower stability the bonds formed by condensation reactions, which can undergo hydrolysis in an aquatic environment. With a value above $400 \mathrm{M} \Omega$, the average leak impedance of the failed channels at the end of the measurements is an order of magnitude larger in the $\mathrm{PI}-2$ version with respect to the PI-1 version, and could be acceptable for many applications.

The clear improvement of leak impedance can also be seen in figure 6, which shows the average impedance (average taken over all channels of a certain type) as a function of time in saline solution.

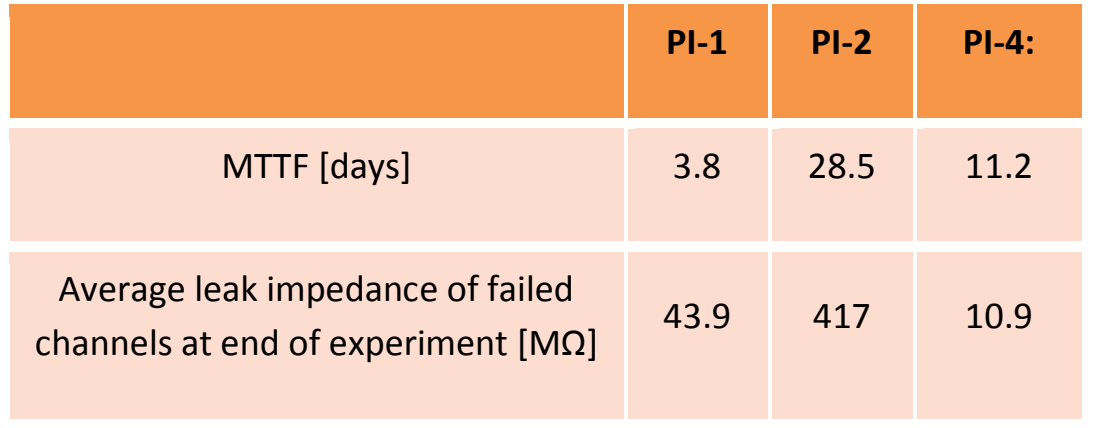

Table 2: measured lifetimes at $40^{\circ} \mathrm{C}$ and average leak impedance at end of experiment 
Another observation was that the reduction of impedance was permanent: even after drying for 3 weeks in air, channels that had fallen below $50 \mathrm{M} \Omega$ did not return to higher values when re-immersed in PBS.

Another failure mode, the breaking of conductive paths, was assessed by monitoring for a strong rise in DC impedance (i.e. to a value higher than $200 \mathrm{M} \Omega$ ) between the conductive paths ending on an electrodes and a stainless steel reference electrode in the PBS. No breaks were detected over the test period in any of the samples.

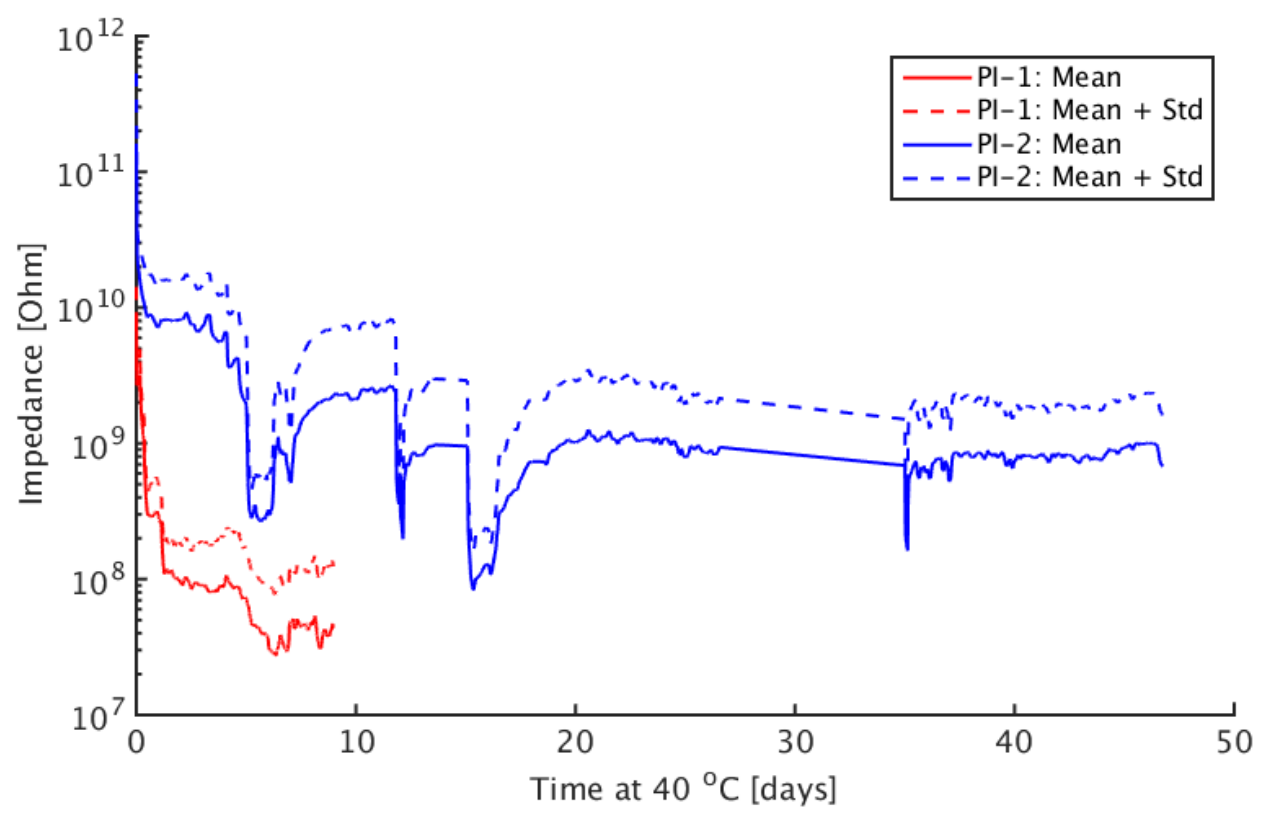

Figure 6: plot of the average leak impedance over 7 channels as a function of time in saline solution at 40 ${ }^{\circ} \mathrm{C}$. The order of magnitude improvement offered by the PI-2 process over the standard PI-1 process is clearly visible. Dotted lines denote the standard deviation.

\subsection{Comparison to Parylene-C}

Parylene-C test samples, $12.5 \mu \mathrm{m}$ thick, were fabricated using the same masks as the PI samples. The samples had to be made thicker as $7 \mu \mathrm{m}$ thick Parylene- $C$ samples proved to curl up too much as be impractical to handle. In order to improve insulation performance [15], the samples were annealed in a nitrogen atmosphere at $200^{\circ} \mathrm{C}$ for 24 hours, restrained between two Teflon sheets. In order to compare lifetimes in a short timeframe, both PI-2 samples as well as Parylene-C samples were then put in PBS at accelerated aging conditions $\left(87^{\circ} \mathrm{C}\right)$, each with at least 7 channels. No difference in lifetime - defined the same as above - was found $(p=0.95)$ at the accelerated aging temperature. 


\section{Discussion}

The presented data adds arguments to the discussion on the insulation material of choice for thin film neural implants.

Stieglitz et al. [21] already showed that polyimides such as PI-2611 are mechanically superior compared to Parylene C: PI-2611's tensile strength of $390 \mathrm{MPa}$ is almost a factor 6 higher than Parylene C's. Furthermore, its Young's modulus is about 2.6 times higher. This translates into much higher bendability for implants of comparable thickness. Another advantage is the polyimide's thermal expansion coefficient $(3 \mathrm{ppm} / \mathrm{K})$ which approaches silicon's. This translates in negligible bending of the polyimide after release from the silicon substrate.

These superior properties were also clear in our own experimentation beyond the scope of this paper: Parylene $C$ implants had to be made $80 \%$ thicker ( 12.5 instead of $7 \mu \mathrm{m}$ ) in order to have a manageable bending after release and to allow handling the released implants without too much risk of breaking. Still, they were found to be relatively brittle while the $7 \mu \mathrm{m}$ thick PI implants could withstand significant abuse while being more flexible.

However, what was until now not clear from literature is the very limited insulation lifetime of PI in a saline environment when it is applied using the standard fabrication process. Actually, the lifetime is only useful for acute measurements, lasting a few days at most. In this work, we have shown two improved fabrication processes to improve the insulation lifetime. The best process, $\mathrm{PI}-2$, improves the lifetime of the test structures with a factor of 7.5. Also, with an average leak impedance that is $10 \mathrm{x}$ higher than that of PI-1 test structures at the end of the experiment, most channels fabricated with this method remain useful in a lot of measurement applications even after our failure criterion has been reached.

Nevertheless, recent work has shown that very high annealing temperatures $\left(300^{\circ} \mathrm{C}\right) \mathrm{can}$ improve the reliability of Parylene insulation even higher, reaching over one year in saline solution at $37{ }^{\circ} \mathrm{C}$ [16]. If such high annealing temperatures are compatible with the rest of the fabrication process, Parylene is still the preferred choice for achieving the highest insulation lifetime. The rest of the fabrication process needs to be considered as well indeed: in particular the adhesion of the other layers to be deposited, the intrinsic stresses of those materials should be considered and of course the mechanical behavior of the material should be acceptable for the application.

Another argument is the biocompatibility. Both materials have demonstrated excellent biocompatibility in a series of in vivo studies. However, PI2611 is currently not certified for use in implants in humans under ISO10993 or USP classification [21], whereas Parylene-C is already in use in e.g. cochlear implants. This is of course not an argument when designing implants for animal studies. However, researchers wanting their implants to be eventually used in humans should consider the significant extra cost of convincing a supplier to get the certification if this can be done at all or, alternatively, an own formulation of polyimide. 


\section{Conclusion}

In conclusion, there are still situations in which polyimide is preferable over Parylene and vice versa; what is the best choice depends on the expected circumstances of use and the rest of the fabrication process. Polyimide is superior in terms of mechanical properties and ease of fabrication while Parylene$\mathrm{C}$, annealed at $300{ }^{\circ} \mathrm{C}$, still seems to have the highest insulation lifetime. By improving the lifetime and insulation impedance of polyimide insulation with one order of magnitude, this work has helped to make the applicability of polyimide in chronic thin film neural interfaces considerable headway.

\section{Acknowledgements}

This research was only made possible by Frederik Ceyssens' research fellowship from FWO-Flanders, the KULeuven IDO fund and further supported by the Hercules Foundation for heavy equipment (AKUL 034 and ZW1115). The research leading to these results has received funding from the European Research Council under the European Union's Seventh Framework Programme (FP7/2007-2013) / ERC grant agreement $n^{\circ}$ 340931. Also, special thanks to Tony Van Nuland for the FIB imaging work and Noella Gaethofs for bonding the devices.

\section{References}

1. HajjHassan M, Chodavarapu V, Musallam S 2008 NeuroMEMS: neural probe microtechnologies., Sensors 8(10): 6704-6726.

2. Chao Z, Nagasaka Y, Fujii N 2010 Long-term asynchronous decoding of arm motion using electrocorticographic signals in monkeys. Frontiers in neuroengineering 3.

3. Boppart S A, Wheeler B C and Wallace C S 1992 A flexible perforated microelectrode array for extended neural recordings IEEE Trans. Biomed. Eng. 39 37-43

4. Owens A L, Denison TJ, Versnel H, Rebbert M, Peckerar M, Shamma S A 1995 Multi-electrode array for measuring evoked potentials from surface of ferret primary auditory cortex, Journal of Neuroscience Methods 58 209-220

5. Stieglitz T, Beutel H, Schuettler M and Meyer J-U 2000 Micromachined, polyimide-based devices for flexible neural interfaces Biomed. Microdevices 2 283-94

6. Rubehn B, Bosman C, Oostenveld R, Fries P and Stieglitz T 2009 A MEMS-based flexible multichannel ECoG-electrode array, J. Neural Eng. 6 ( 036003

7. Kim D, Viventi J, Amsden J, Xiao J, Vigeland L, Kim Y, Blanco J, Panilaitis B, Frechette E, Contreras, D et al. 2010 Dissolvable films of silk fibroin for ultrathin conformal bio-integrated electronics. Nature materials 9 (6) 511517

8. Mokwa W 2004 MEMS technologies for epiretinal stimulation of the retina, Journal of Micromechanics and Microengineering 14(9) S12-S16.

9. Shamma-Donoghue S A, May G A, Cotter N E, White R L and Simmons F B 1982 Thin-film multielectrode arrays for a cochlear prosthesis IEEE Trans. Electron. Dev.29 136-44

10. Sterken T, Vanfleteren J, Torfs T, Op de Beeck M, Bossuyt F, Van Hoof C 2011 Ultra-Thin Chip Package (UTCP) and Stretchable Circuit Technologies for Wearable ECG System, In $33^{\text {rd }}$ annual international conference of the IEEE EMBS, pp. 6886-6889. 
11. Yeager, J. D., Phillips, D. J., Rector, D. M., \& Bahr, D. F. 2008 Characterization of flexible ECoG electrode arrays for chronic recording in awake rats. Journal of neuroscience methods, 173(2), 279-285.

12. Polikov V, Tresco P, Reichert W 2005 Response of brain tissue to chronically implanted neural electrodes. Journal of neuroscience methods 148 (1), 118.

13. Henle C, Raab M, Cordeiro J G, Doostkam S, Schulze-Bonhage A., Stieglitz T, Rickert J. 2011 First long term in vivo study on subdurally implanted Micro-ECoG electrodes, manufactured with a novel laser technology. Biomedical microdevices, 13(1), 59-68.

14. Ordonez J S, Boehler C, Schuettler M and Stieglitz T 2012 Long-term adhesion studies of polyimide to inorganic and metallic layers, Mater. Res. Soc. Proc. 1466 7-13.

15. Rodger D C et al. 2008 Flexible parylene-based multielectrode array technology for high-density neural stimulation and recording, Sensors and Actuators B: chemical 132(2) 449-460.

16. Von Metzen R P and Stieglitz T 2013 The effects of annealing on mechanical, chemical, and physical properties and structural stability of Parylene C, Biomed Microdevices 15 727-735

17. Ledochowitsch P, Tiefenauer R F, Pepin B, Maharbiz M. M and Blanche T. J. 2013 Nanoflex for neural nanoprobes. In Solid-State Sensors, Actuators and Microsystems (TRANSDUCERS \& EUROSENSORS XXVII), 2013 Transducers \& Eurosensors XXVII: The 17th International Conference on (pp. 1278-1281). IEEE.

18. Ceyssens F, van Kuyck K, Velde G, Welkenhuysen M, Stappers L, Nuttin B and Puers R 2013 Resorbable scaffold based chronic neural electrode arrays, Biomedical Microdevices 15 481-493.

19. Liston E M, Martinu L and Wertheimer M R 1993 Plasma surface modification of polymers for improved adhesion: a critical review, Journal of Adhesion Science and Technology 7 1091-1127.

20. Stoffel N C, Hsieh M, Chandra S, and Kramer E J 1996 Surface Modification Studies of Polyimide Films Using Rutherford Backscattering and Forward Recoil Spectrometry Chem. Mater. 8, 1035-1041.

21. Stieglitz T, Beutel H, Schuettler M and Meyer J 2000 Micromachined, Polyimide-Based Devices for Flexible Neural Interfaces, Biomedical Microdevices 2:4 283-294.

22. Hassler C, Boretius T, Stieglitz T 2011 Polymers for Neural Implants, Journal of Polymer Science: part b: Polymer Physics 49, 18-33. 